\title{
First-Principles Study of the Ferromagnetic and Semiconductor Properties of $\left[\mathrm{Mn}\left(4,4^{\prime}-\text { bipy }\right)\left(\mathrm{N}_{3}\right)_{2}\right]_{n}$
}

\author{
J.W. $\mathrm{Li}^{1}$, G.Y. Gao ${ }^{2}$, and F. $\mathrm{Jia}^{3}$ \\ ${ }^{1}$ Department of Physics, Nanchang Institute of Technology, Nanchang 330099, China \\ ${ }^{2}$ Department of Physics, Huazhong University of Science and Technology, Wuhan 430074, China \\ ${ }^{3}$ Department of Physics, Jiangxi Normal University, Nanchang 330022, China
}

Received on 11 September, 2007

\begin{abstract}
Based on density functional theory, the full potential linearized augmented plane wave (FPLAPW) method is applied to study the electronic structure and the magnetic properties of the compound $\left[\mathrm{Mn}\left(4,4^{\prime}-\mathrm{bipy}\right)\left(\mathrm{N}_{3}\right)_{2}\right]_{n}$. The density of states, the spin magnetic moment and the electronic band structure are calculated. The results indicate that the compound has ferromagnetic and semiconductor properties. It is found that there is hybridization between the $\mathrm{d}$ orbital of the central $\mathrm{Mn}$ atom and the $\mathrm{p}$ orbital of the $\mathrm{N}$ atom, and that there exists a spin delocalization from the $\mathrm{Mn}$ atom towards the $\mathrm{N}$ atoms of the EE-azide groups. Through the EE-azide bridges, the spin delocalization makes the neighboring $\mathrm{Mn}$ atoms having a dominant ferromagnetic interaction. The magnetic moment $5.0 \mu_{B}$ per molecule mainly comes from $\mathrm{Mn}$ ion with little contribution from the EE-azide groups.
\end{abstract}

Keywords: FPLAPW; Electronic structure; Ferromagnetic; Semiconductor

\section{INTRODUCTION}

In the past decades, the molecular-based magnetic materials with paramagnetic metal ions, where the bridging ligands act as a super-exchange pathway between the metal centers, have been considerably studied both experimentally and theoretically, and some new materials have also been synthesized [1-5]. Recently the investigation of organic magnetic materials containing only light elements, typically $\mathrm{C}, \mathrm{H}, \mathrm{O}, \mathrm{N}$ and $\mathrm{S}$, has been drawn more and more attention [6-11]. Pure organic magnetic materials have free radicals, which can supply the magnetic moment, but generally their transition temperatures are too low for practical applications. The synthesis of the molecular-based ferromagnet with metallic ions has attracted an extensive interest. The solid-state assembly of metal-organic polymers has also the potential applications in nonlinear optics, magnetism and molecular recognition.

The compound $\left[\mathrm{Mn}\left(4,4^{\prime} \text {-bipy }\right)\left(\mathrm{N}_{3}\right)_{2}\right]_{n}$ consists of a 3D network in which $\mathrm{Mn}$ (II) cations are octahedrally coordinated to six $\mathrm{N}$-atoms: four of them corresponding to single $\mathrm{EE}$ azides, on the equatorial positions, and two of them to 4,4'-bipy ligands, on the axial ones $[12,13]$. The structure is rather complex as the six ligands connected to each metal ion perform as intermetallic bridges. The pseudohalide ligands provide a tetrahedral topology around $\mathrm{Mn}$ (II) cations resulting in an azide-linked diamondoid network, which have been recognized as potential structural motifs for the construction of acentric solids. The singularity of the compound lies on the absence of a center of symmetry that is a requirement for many bulk properties of solid materials. The structure of the compound $\left[\mathrm{Mn}\left(4,4^{\prime} \text {-bipy }\right)\left(\mathrm{N}_{3}\right)_{2}\right]_{n}$ with atom numbering scheme is shown in Fig. 1. According to the susceptibility measurement in experiment, the present complex is ferromagnetic at low temperatures [13].

In order to understand well the magnetic and electronic properties of the compound $\left[\mathrm{Mn}\left(4,4^{\prime}-\text { bipy }\right)\left(\mathrm{N}_{3}\right)_{2}\right]_{n}$, in the present paper, we report our accurate first-principles calcu-

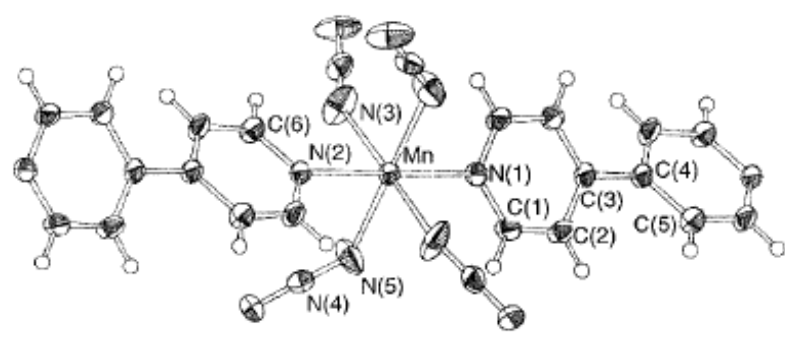

FIG. 1: ORTEP diagram of $\left[\mathrm{Mn}\left(4,4^{\prime} \text {-bipy }\right)\left(\mathrm{N}_{3}\right)_{2}\right]_{n}$ with atom numbering scheme showing $50 \%$ probability ellipsoids [14].

lations. To our knowledge this is the first attempt based on molecular entity for this compound theoretically. The main points of such work are to investigate the electronic structure, the magnetic coupling, and to analyze the propagation of the observed ferromagnetic interaction via the density of states (DOS) and the electronic band structure. This provides new insights into the origin of the ferromagnetic coupling in the compound which should be useful for the design of novel molecular-based ferromagnet with metallic ions. Our results show that there exists a dominant ferromagnetic interaction arises from the EE-azide groups part of the compound. In addition, we find that the spin magnetic moment mainly comes from $\mathrm{Mn}$ ion, and there are smaller positive spin populations on the EE-azide groups. This paper is organized as follows. In Section 2 we present the computational method. Section 3 is devoted to the results and discussions. Finally, we summarize the results in Section 4.

\section{COMPUTATIONAL METHODS}

The calculations presented in this work are performed by employing the first principles full potential linearized augmented plane wave (FPLAPW) method, which is among the 
most accurate band structure methods presently available and has been applied to materials simulation successfully. In this method, no shape approximation is used for either the potential or the electronic charge density. We use the wien2k [14] package that allows inclusion of local orbitals in the basis, which upon linearization improves and makes possible a consistent treatment of semi-core and valence in one energy window. In general, the local density approximation (LDA) and the generalized gradient approximation (GGA) can be chosen in wien $2 \mathrm{k}$ code. Considering the success of the GGA derived from their ability to correct some LDA deficiencies with a modest increase in computational workload, the GGA is mandatory for systems containing "weak" or hydrogen bonds such as molecular crystals; for these systems the intermolecular bond lengths are severely underestimated in LDA, but they can be appreciably improved in GGA. In addition, the GGA has been demonstrated to be capable of calculating organic magnets [15]. So in our calculations, the exchange and correlation effects are treated with the GGA according to Perdew et al [16].

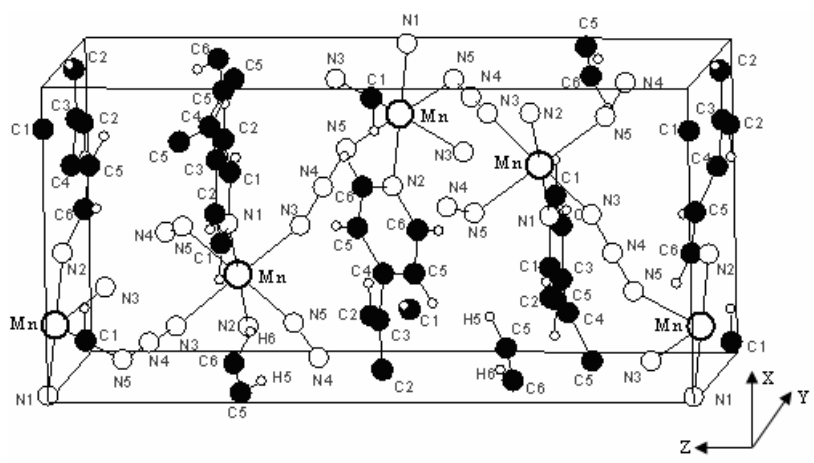

FIG. 2: ORTEP labeled primitive cell for $\left[\mathrm{Mn}\left(4,4^{\prime}-\text { bipy }\right)\left(\mathrm{N}_{3}\right)_{2}\right]_{n}$. (For clarity we only label the hydrogen atom in part.)

The compound $\left[\mathrm{Mn}\left(4,4^{\prime} \text {-bipy }\right)\left(\mathrm{N}_{3}\right)_{2}\right]_{n}$ crystallizes in the tetragonal system, space group is P43212. There are four molecules in one primitive cell, as shown in Fig. 2. For clear view, we only label the hydrogen atom in part. The structure parameters of the compound are $a=8.229(2) \AA, b=8.229(2) \AA$, $\mathrm{c}=16.915(7) \AA, \alpha=\beta=\gamma=90^{\circ}$. The atomic-sphere radii $R_{m t}$ are chosen as $2.18,1.08,1.13$ and 0.61 a.u. for $\mathrm{Mn}, \mathrm{N}, \mathrm{C}$ and $\mathrm{H}$, respectively. We set the charge density Fourier expansion up to $\mathrm{l}=10$ and the charge density Fourier expansion cutoff $G_{\max }=14$ in the muffintins. One hundred $k$-points in the first Brillouin zone are adopted in the calculations. The plane wave cutoff energy is $146 \mathrm{eV}$. In order to achieve a satisfactory degree of convergence, the cutoff parameter is taken to be $R_{m t} K_{\max }=2.0$, where $K_{\max }$ is the maximal value of the reciprocal lattice vector used in the plane wave expansion, and $R_{m t}$ is the smallest atomic sphere radius in the unit cell. The selfconsistency calculations are considered to be converged only when the energy difference between input and output energy is less than $10^{-4}$ Ryd per formula unit.

\section{RESULTS AND DISCUSSION}

In order to study the electronic structure and the magnetic properties of $\left[\mathrm{Mn}\left(4,4^{\prime} \text {-bipy }\right)\left(\mathrm{N}_{3}\right)_{2}\right]_{n}$, the total DOS of the molecule, the total DOS of Mn, N3, N4, N5 are calculated as shown in Fig. 3. Because the DOS distribution near the Fermi level determines the magnetic properties, we concentrate our attention on the DOS distribution from -6 to $2 \mathrm{eV}$. In the vicinity of the Fermi level, we find that the total DOS distribution of the up- and down-spin electrons is obviously split. These energy bands are decomposed into two subbands: one is the up-spin valence band; the other is the down-spin band. Thus the ordered spin arrangement of electrons is formed by the exchange correlation of electrons, which provides the static magnetic moment of this compound. According to the DOS distribution, it is worthwhile to note that the main origin of magnetism is provided by $\mathrm{Mn}$ atom, in addition, the N3, N4 and N5 atoms also contribute to the spin magnetic moment, and the net spin of $\mathrm{Mn}$ is parallel to those of N3, N4 and N5. These phenomena play an important role in the indirect interaction between $\mathrm{Mn}(\mathrm{II})$ ions, which implies that the four EE-azide ligands act as super-exchange pathway. It is more interesting that near the Fermi level, the top of the occupied spin-up band is $1.0 \mathrm{eV}$ below the Fermi level. There is an energy gap of $1.17 \mathrm{eV}$ between the spin-up occupied subband and the spin-up unoccupied subband. While the total spindown DOS is above the Fermi level. So the compound has semiconductor properties.

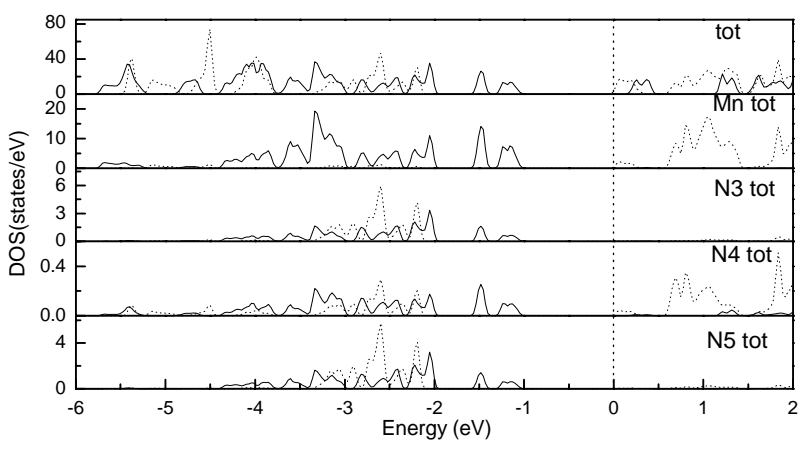

FIG. 3: DOS of the molecule and Mn, N3, N4, N5 atoms (solid: spin-up states; dotted: spin-down states. The Fermi level is located at $0 \mathrm{eV}$ ).

Now, let us turn our attention to the spin magnetic moments on atoms, which are defined as the difference of the average number of occupied ions with spin-up and spin-down in the muffin-tin sphere. It can be seen from Table 1, The Mn atom has the largest magnetic moment of $4.311 \mu_{B}$ and the magnetic moment of $\mathrm{N} 1, \mathrm{~N} 2, \mathrm{~N} 3, \mathrm{~N} 4$ and $\mathrm{N} 5$ are much larger than that of the other atoms. The magnetic moment calculations show that the major contribution of the spin magnetic moments is from $\mathrm{Mn}$ atom, and there are smaller positive spin populations on the nitrogen atoms. This phenomenon is the result of spin delocalization from the $\mathrm{Mn}$ atom towards the $\mathrm{N}$ atoms. In addition, the magnetic moment of $\mathrm{N} 1$ and $\mathrm{N} 2$ is much smaller compared with that of N3, N4 and N5. It suggests that the 
TABLE I: Atomic spin magnetic moments of $\left[\mathrm{Mn}\left(4,4^{\prime}-\text { bipy }\right)\left(\mathrm{N}_{3}\right)_{2}\right]_{n}$

\begin{tabular}{|c|c|c|c|}
\hline Site & Magnetic moment $\left(\mu_{B}\right)$ & Site & Magnetic moment $\left(\mu_{B}\right)$ \\
\hline Mn1 & 4.31100 & C2 & 0.00033 \\
\hline N1 & 0.00858 & H2 & 0.00007 \\
\hline N2 & 0.00888 & C3 & 0.00139 \\
\hline N3 & 0.02145 & C4 & 0.00094 \\
\hline N4 & 0.01148 & C5 & 0.00050 \\
\hline N5 & 0.02043 & H5 & 0.00007 \\
\hline C1 & 0.00261 & C6 & 0.00211 \\
\hline H1 & 0.00004 & H6 & 0.00005 \\
\hline
\end{tabular}

spin delocalization from the Mn atom towards the two 4,4'bipy ligands is weak. That is to say, the ferromagnetic interaction mainly arises from the four EE-azide bridges. In the present calculations, the spin magnetic moment of the unit cell is $20.004 \mu_{B}$. Because there are four molecules in one primitive cell, the spontaneous magnetic moment of the molecule is about $5.001 \mu_{B}$ per molecule. The $\mathrm{Mn}^{2+}$ ion with $3 \mathrm{~d}^{5}$ has five unpaired electron, therefore our calculations is reasonable. In the FPLAPW method, the unit cell is divided into two parts: (1) non-overlapping atomic spheres (centered at the atomic sites) and (2) an interstitial region. The magnetic moments of atoms add up to a saturation moment of $4.390 \mu_{B}$ per molecule, and the interstitial moment yields the remaining $0.699 \mu_{B}$ per molecule.

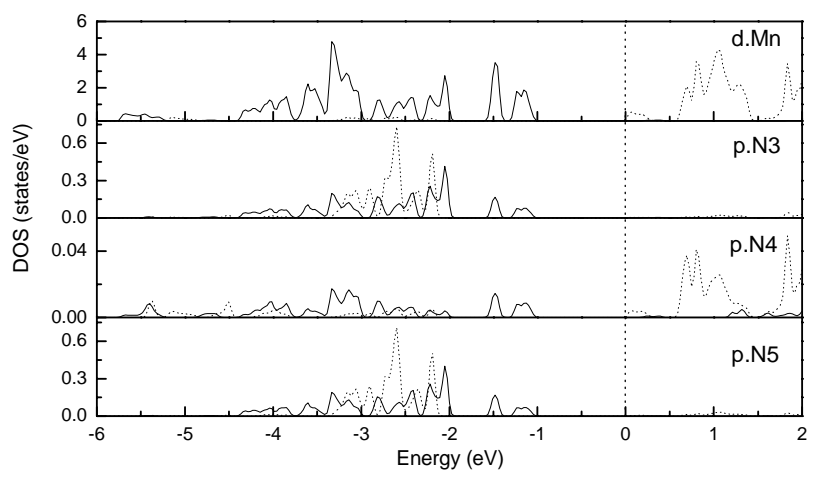

FIG. 4: The calculated partial density of states (PDOS) for Mn, N3, N4, N5 atoms (solid: spin-up states; dotted: spin-down states. The Fermi level is located at $0 \mathrm{eV}$ ).

To exploit the mechanism of the magnetic interactions in $\mathrm{Mn}\left(4,4^{\prime} \text {-bipy) }\left(\mathrm{N}_{3}\right)_{2}\right]_{n}$, the partial density of states of $\mathrm{Mn} \mathrm{d}, \mathrm{N} 3$ $\mathrm{p}, \mathrm{N} 4 \mathrm{P}$ and N5 p is shown in Fig. 4. It is quite obvious that the total atomic DOS comes nearly only from d orbitals of Mn and p orbitals of N3, N4, N5 in the energy range. In addition, the partial DOS of Mn d, N3 p, N4 P and N5 p below the Fermi level have similar peaks and character, which indicates that there exist hybridizations between Mn d and N3 p, N4 P and $\mathrm{N} 5 \mathrm{p}$. It is worthwhile to say that the hybridizations reveals two points: (i) the four orbitals are strongly mixed over the energy region which means the $\mathrm{N} 3 \mathrm{P}$ orbital, N4 P orbital and the N5 P orbital are responsible for the magnetic coupling in

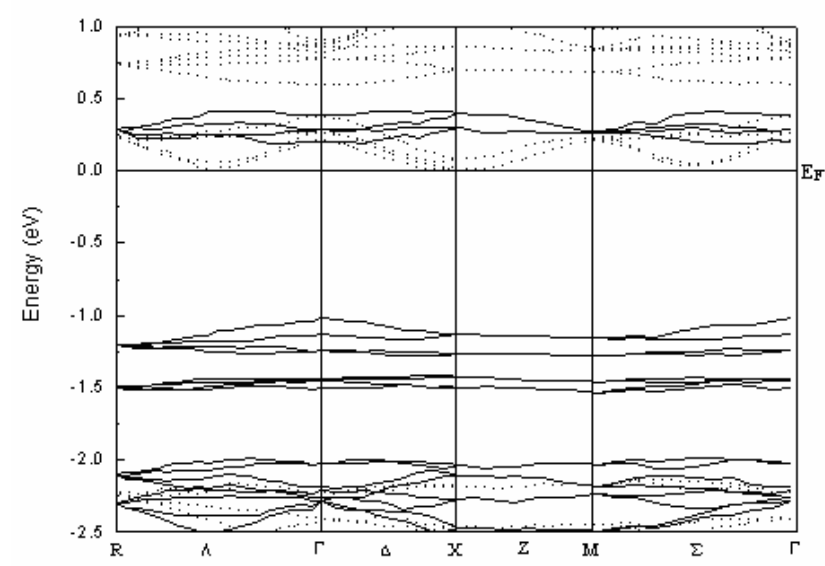

FIG. 5: Electronic bands of $\left[\mathrm{Mn}\left(4,4^{\prime} \text {-bipy }\right)\left(\mathrm{N}_{3}\right)_{2}\right]_{n}$ along selected symmetry lines within the first Brillouin zone. The horizontal solid line denotes the Fermi level. The solid lines denote the spin-up electronic band structure. The dotted lines denote the spin-down electronic band structure.

the molecule, and the strong overlap of the magnetic orbitals below the Fermi level between Mn and the four EE-azide ligands that link the manganese centers indicates the contribution of the super-exchange interaction to the magnetism and (ii) the net spin of $\mathrm{Mn} \mathrm{d}$ is parallel to those of N3 p, N4 P and N5 p, so the ordered spin arrangement is formed by the super-exchange interaction.

For further consideration, we also give the electronic band structure in Fig. 5 (solid line is the spin-up electronic band; dashed line is the spin-down electronic band). As shown in Fig. 5, the spin-up electronic bands are below the Fermi level, but the spin-down electronic bands are above the Fermi level. This means that there exists a ferromagnetic interaction between the molecules. Furthermore, we can see that the top of the spin-up occupied bands is $1.0 \mathrm{eV}$ below the Fermi level and there is an energy gap of $1.17 \mathrm{eV}$ between the spin-up occupied bands and the spin-up unoccupied bands, which implies that the complex has the properties of a semiconductor.

\section{CONCLUSIONS}

The magnetic and the semiconductor properties have been studied by employing the FPLAPW within the framework of density functional theory for the compound [Mn $\left(4,4^{\prime}\right.$ bipy) $\left.\left(\mathrm{N}_{3}\right)_{2}\right]_{n}$. The results show that the compound has ferromagnetic and semiconductor properties at low temperature. The analysis of the DOS and the electronic band structure reveals that the spin magnetic moment is $5.0 \mu_{B}$ per molecule and the magnetic moment is mainly from the $\mathrm{Mn}$ ion and a little contribution from the EE-azide groups. It is also found that there exists a dominant ferromagnetic interaction arising from the EE-azide groups part of the compound. 


\section{Acknowledgments}

We are grateful to Drs. L.Y. Hu, and J. Zhang for their valuable discussions.
[1] J. Ribas, M. Monfort, C. Diaz, C. Bastos, and X. Solans, Inorg. Chem. 33, 484 (1994).

[2] L. Deakin, M.A. Arif, and J.S. Miller, Inorg. Chem. 38, 5072 (1999).

[3] A. Escuer, R. Vicente, M.A.S. Goher, and F.A. Mautner, Inorg. Chem. 35, 6386 (1996).

[4] E. Bakalhassis, P. Bergerat, O. Kahn, S. Jeannin, Y. Jeanin, Y. Dromzee, and M. Guillot, Inorg. Chem. 31, 625 (1992).

[5] A. Escuer, R. Vicente, J. Ribas, and X. Solans, Inorg. Chem. 34, 1793 (1995)

[6] Z. Fang, Z.L. Liu, K.L. Yao, and Z.G. Li, Phys. Rev. B 51, 1304 (1995).

[7] Y.F. Duan and K.L. Yao, Phys. Rev. B 134, 6313 (2001).

[8] W.Z. Wang, J. Chem. Phys. 108, 2867 (1998).

[9] T. Sugano, S.J. Blundell, W. Hayes, and P. Day, Synth. Metals 121, 1812 (2001).

[10] S.J. Luo and K.L. Yao, J. Magnet. Magnet. Mater 257, 11
(2003).

[11] W.Z. Wang, Z.L. Liu, and K.L. Yao, Phys. Rev. B 55, 12989 (1997).

[12] S. Han, J.L. Manson, J. Kim, and J.S. Miller, Inorg. Chem. 39, 4182 (2000).

[13] S. Martín, M.G. Barandika, L. Lezama, J.L. Pizarro, Z.E. Serna, J.I.R.D. Larramendi, M.I. Arriortua, T. Rojo, and R. Cortés, Inorg. Chem. 40, 4109 ( 2001).

[14] P. Blaha, K. Schwarz, G.K.H. Madsen, D. Kvasnicka, J. Luitz, Wien2k, Vienna University of Technology, 2002, improved and updated Unix version of the original copyrighted Wien-code, which was published by P. Blaha, K. Schwarz, P. Sorantin, and S.B. Trickey, Comput. Phys. Commun. 59, 399 (1990).

[15] L. Ducase and A. Fritsh, Chem. Phys. Lett. 286, 183 (1998).

[16] J.P. Perdew, S. Burke, and M. Ernzerhof, Phys. Rev. Lett. 77, 3865 (1996). 